\title{
Non-intubated (tubeless) uniportal video-assisted thoracoscopic lobectomy
}

\author{
Diego Gonzalez-Rivas ${ }^{1,2 *}$, Yang Yang $^{2 *}$, William Guido ${ }^{2}$, Gening Jiang ${ }^{2}$ \\ ${ }^{1}$ Department of Thoracic Surgery, Coruña University Hospital and Minimally Invasive Thoracic Surgery Unit (UCTMI), Coruña, Spain; \\ ${ }^{2}$ Department of Thoracic Surgery, Shanghai Pulmonary Hospital, Tongji University School of Medicine, Shanghai 200433, China \\ *These authors contributed equally to this work. \\ Correspondence to: Diego Gonzalez-Rivas, MD, FECTS. Department of Thoracic Surgery, Coruña University Hospital, Xubias 84, 15006, Coruña, \\ Spain. Email: diego.gonzalez.rivas@sergas.es.
}

Submitted Nov 24, 2015. Accepted for publication Jan 28, 2016.

doi: $10.21037 /$ acs.2016.03.02

View this article at: http://dx.doi.org/10.21037/acs.2016.03.02

During the last decade, there have been important developments in new minimally invasive surgical techniques for major pulmonary resections (1). Advances in anesthesiology include a lack of general anesthesia in thoracic operations, by maintaining spontaneous ventilation, and minimal sedation of patients. The choice of a single-incision technique combined with the lack of general anesthesia further minimize the invasiveness of the procedure (2). Moreover, a 'tubeless' concept can be perfectly applied to these procedures. This involves the complete absence of a tracheal tube or even laryngeal mask, central line, urinary catheter or epidural. We present a video showing three cases of non-intubated (tubeless) uniportal video-assisted thoracoscopic surgery (VATS) major pulmonary resections (Video 1).

\section{Clinical vignettes}

\section{First case}

The patient is a 50 year-old female with a $4 \mathrm{~cm}$ adenocarcinoma located on the right upper lobe (RUL) with no lymph node involvement. The video shows a right upper lobectomy.

\section{Second case}

The patient is a 60 year-old male with a $3 \mathrm{~cm}$ tumor on the RUL. After a right upper lobectomy, a complete paratracheal lymph node dissection was performed, as shown in the video.

\section{Third case}

The patient is a 68 year-old male with a $4.2 \mathrm{~cm}$ non-small cell lung cancer in the left upper lobe with adhesions to the chest wall. The video shows a left upper lobectomy and lymph node dissection.

\section{Clinical preparation}

All patients underwent standard monitoring including electrocardiography, non-invasive blood pressure, pulse oximetry and respiratory rate measurements, with an approximation of the end-tidal carbon dioxide with a catheter placed in one nostril. The adequacy of ventilation and depth of sedation was monitored by using bispectral index or entropy.

The pharmacological management was based on a target-controlled infusion of remifentanyl and propofol, with a premedication of midazolam $(0.15-0.25 \mathrm{mg} / \mathrm{kg})$ and atropine $(0.01 \mathrm{mg} / \mathrm{kg}) 15$ minutes before anaesthesia, adjusting real-time rate of infusion with the aggressiveness of each period during the surgery.

As an intercostal nerve block, local anesthetic was administered at level T4-T5, in order to avoid any reaction of the patient due to the surgical incision (cases 2 and 3). For case 1 we only used a thoracoscopic intercostal block infiltration mixture of lidocaine $1 \%$ and levobupivacaine $0.2 \%$ to ensure fast onset of action and prolonged analgesia. We performed intrathoracic vagal blocks to inhibit visceral irritation and to minimize unwanted cough responses during nonintubated VATS (levobupivacaine $2 \mathrm{~mL}, 0.25 \%)$. Oxygen (6-9 L/min) was supplied via facial 
mask during the surgery. No central line, urinary catheter or epidural were used during the procedures.

\section{Clinical results}

All patients had an excellent recovery and were discharged home on the third, second and fourth postoperative days respectively with no complications.

\section{Advantages}

One of the major advantages of non-intubated VATS is to offer surgical opportunities for patients who are too risky for intubated general anesthesia A permissible transitory hypercapnia was usually noted in patients with severe emphysema but resolved after procedures

The quality of the pulmonary collapse obtained by iatrogenic pneumothorax via VATS under spontaneous breathing is excellent, or at least as good as in mechanical ventilation using a double-lumen endotracheal tube or bronchial blocker. The mechanism of performing this lung collapse is more physiological via a small intercostal incision than via one-lung ventilation (OLV), resulting in less lung inflammation and stress with the possibility of a faster and improved postoperative recovery and outcome (3).

Single-lung ventilation under spontaneous breathing also preserves a better match of ventilation and perfusion in the surgical position and avoids intubation-related airway trauma, mechanical ventilation-induced lung injury, residual muscular blockade and postoperative nausea and vomiting. Respiratory efficiency is increased by diaphragmatic function, which is maintained. Intrapulmonary shunting and hypoxemia are reduced compared to OLV intubated patients (4).

Another advantage of maintaining negative intrathoracic pressure due to spontaneous breathing is improved hemodynamic control, with no decrease in venous return related to the positive intrathoracic pressure generated during mechanical ventilation. Data from preliminary studies also suggest an attenuated stress response after awake VATS in comparison with equivalent procedures performed under general anesthesia and one-lung mechanical ventilation $(3,4)$.

\section{Caveats}

Current reported studies support the contraindication of awake uniportal VATS in patients with anticipated difficulties in airway management, obesity (body mass index >30), dense and extensive pleural adhesions, hemodynamic instability, ASA classification over II and tumours greater than $6 \mathrm{~cm}$ (5). In order to ensure patient safety, a clearly defined protocol for elective or urgent intubation must be determined prior to the operation. Sometimes, intraoperative conversion to general anesthesia is inevitable and the surgical team must have a plan to minimize the risk of the patient. In case of complications such as major bleeding, strong adhesions, significant mediastinal movement or persistent hypoxaemia and tachypnea, it is suggested to make an early conversion to intubated general anaesthesia. We recommend the insertion of a doublelumen tube maintaining the lateral decubitus position for expert and skilled anaesthesiologists or, if it is not possible, intubation with a single-lumen tube followed by insertion of a bronchial blocker while trying to maintain the patient's position.

There is a risk of experiencing a hypercapnia-related rebreathing effect from the initial paradoxical respiration and hypoventilation due to collapse of the operated lung and sedation. However, it is rare to need support ventilation, which is only needed in patients with a severe restrictive or obstructive ventilatory defect. Ventilatory obstruction and hyperinflation in a dependent ventilated lung produce intrinsic positive end-expiratory pressure and decrease mediastinal shift, thus increasing functional residual capacity (FRC) and decreasing atelectasis. This reduces the risk of hypoxemia contrary to the restrictive ventilatory defect (4).

\section{Conclusions}

Nowadays, the evolution of video-assisted thoracic surgery to less invasive techniques, such as uniportal VATS (with only one incision of $3 \mathrm{~cm}$ ) allows us to reconsider the possibility of avoiding intubation with general anesthesia for lobectomy. However, these non-intubated major pulmonary resections must only be performed by very experienced uniportal thoracoscopic surgeons, preferably skilled and experienced in complex or advanced cases and bleeding control through uniportal VATS. When there is a larger series of patients undergoing non-intubated VATS resections, a gold-standard technique may be defined. As a result, most patients could bypass intensive care units and be discharged home early, with a lower incidence of both surgical and anaesthetic complications. As Sir Winston Churchill said in 1942, “This is not the end. It's not even 
the beginning of the end... it's perhaps, the end of the beginning".

\section{Acknowledgements}

None.

\section{Footnote}

Conflicts of Interest: The authors have no conflicts of interest to declare.

\section{References}

1. Ng CS, Lau KK, Gonzalez-Rivas D, et al. Evolution in surgical approach and techniques for lung cancer. Thorax 2013;68:681

Cite this article as: Gonzalez-Rivas D, Yang Y, Guido W, Jiang G. Non-intubated uniportal video-assisted thoracoscopic lobectomy. Ann Cardiothorac Surg 2016;5(2):151-153. doi: 10.21037/acs.2016.03.02
2. Gonzalez-Rivas D, Aymerich H, Bonome C, et al. From Open Operations to Nonintubated Uniportal Video-Assisted Thoracoscopic Lobectomy: Minimizing the Trauma to the Patient. Ann Thorac Surg 2015;100:2003-5.

3. Gonzalez-Rivas D, Bonome C, Fieira E, et al. Nonintubated video-assisted thoracoscopic lung resections: the future of thoracic surgery? Eur J Cardiothorac Surg 2016;49:721-31.

4. Tacconi F, Pompeo E, Sellitri F, et al. Surgical stress hormones response is reduced after awake videothoracoscopy. Interact Cardiovasc Thorac Surg 2010;10:666-71.

5. Chen KC, Cheng YJ, Hung MH, et al. Nonintubated thoracoscopic lung resection: a 3-year experience with 285 cases in a single institution. J Thorac Dis 2012;4:347-51. 